\title{
Reações Cutâneas de Hipersensibilidade Retardada a Medicamentos: Serão os Testes Epicutâneos Positivos Persistentes?
}

\author{
André Pinho', Luís Santiago', Margarida Gonçalo² \\ 'Interno complementar de Dermatologia e Venereologia, Serviço de Dermatologia, Centro Hospitalar e Universitário de Coimbra, \\ Coimbra, Portugal \\ ${ }^{2}$ Chefe de Serviço, Serviço de Dermatologia, Centro Hospitalar e Universitário de Coimbra; Professor auxiliar de Dermatologia \\ Faculdade Medicina da Universidade de Coimbra, Portugal
}

RESUMO - Introdução: Tal como na dermatite de contacto alérgica, nas reações adversas medicamentosas cutâneas (RAMc) retardadas a hipersensibilidade é considerada permanente. No entanto, enquanto na dermatite de contacto alérgica os testes epicutâneos permanecem positivos por longos períodos de tempo, isto tem sido pouco estudado nas RAMc retardadas medicamentos sistémicos. Objetivo: Avaliar, a longo prazo, a persistência das reações positivas dos testes epicutâneos nas RAMc retardadas a antibióticos betalactâmicos, à clindamicina e à carbamazepina. Métodos: Foram selecionados os medicamentos com maior número de reações positivas em testes epicutâneos (betalactâmicos, clindamicina e carbamazepina) e 64 doentes com história de RAMc retardadas com testes positivos a estes fármacos foram convidados a repetir os testes, pelo menos 2 anos depois dos primeiros. As reações dos novos testes foram comparadas com as dos testes originais. Resultados: Nos 23 doentes que aceitaram participar (10 homens/13 mulheres, idade mediana 51 anos) foram observadas 44 reações positivas nos testes iniciais e 40 (91,1\%) destas mantiveram-se positivas, após uma mediana de 6,5 anos (min. 2,0 - máx. 30,7 anos). Relativamente aos betalactâmicos, $17 / 19$ reações permaneceram positivas com aminopenicilinas (amoxicilina ou ampicilina), 8/8 com isoxazolilpenicilinas (flucloxacilina ou dicloxacilina), $3 / 3$ com benzilpenicilina, $1 / 1$ cefoxitina e $1 / 1 \mathrm{com}$ piperacilina. Os testes com clindamicina mantiveram-se positivos em 5/7 ocasiões. Todos os cinco testes repetidos com carbamazepina foram positivos. Em 62,5\% das reações observou-se a mesma intensidade da reação original, não sendo esta afetada pelo tempo decorrido entre os testes, pela idade ou género dos doentes. Conclusão: Nas RAMc retardadas a vários antibióticos e à carbamazepina, a maioria das reações dos testes epicutâneos permaneceu positiva, após vários anos.

PALAVRAS-CHAVE - beta-Lactâmicos; Carbamazepina; Clindamicina; Erupção por Medicamentos; Hipersensibilidade a Medicamentos; Hipersensibilidade Retardada/induzida quimicamente; Testes Epicutâneos.

\section{Non-Immediate Cutaneous Hypersensitivity Drug Reactions: Are Positive Patch Tests Long-Lasting?}

ABSTRACT - Introduction: Like in allergic contact dermatitis, in non-immediate (NI) cutaneous adverse drug reactions (CADR) delayed hypersensitivity to antibiotics and anticonvulsants is considered to be lifelong. Although in allergic contact dermatitis patch tests remain positive for several years, this has seldom been assessed in the setting of NI-CADR to systemic drugs. Objective: To evaluate the long term behaviour of positive patch test reactions in NI-CADR to beta-lactams, clindamycin and carbamazepine. Methods: The drugs associated with the largest number of positive patch test reactions (beta-lactams, clindamycin and carbamazepin) were selected and 64 patients with history of NI-CADR to these drugs and relevant positive reactions were invited to repeat patch tests, at least 2 years thereafter. New patch test reactions were compared with the original ones. Results: In the 23 patients included in the study (10 males/ 13 females, median age 51 years) there were 44 positive reactions at the first patch tests and 40 (91.1\%) of these remained positive after a median interval of 6.5 years (min. 2.0 max. 30.7 years). Concerning beta-lactams, 17/19 reactions persisted positive for aminopenicillins (amoxicillin or ampicillin), $8 / 8$ for isoxazolyl penicillins (flucloxacillin or dicloxacillin), 3/3 for benzylpenicillin, $1 / 1$ for cefoxitin and $1 / 1$ with piperacillin.

Correspondência: André Pinho

Serviço de Dermatologia - Centro Hospitalar e Universitário de Coimbra

Praceta Prof. Mota Pinto - 3000-075, Coimbra, Portugal

Tel.: + 351239400420
Recebido/Received

28 Dezembro/December 2016

Aceite/Accepted

15 Maio/May 2017 
Reactions with clindamycin remained positive in 5/7 cases. All five patch tests repeated with carbamazepine were positive. In $62.5 \%$ of the tests the same intensity of reaction was observed and was not affected by the time interval between tests, gender or age at performing patch tests. Conclusion: In NI-CADR to several antibiotics and carbamazepine, most of the patch test reactions remained positive after several years.

KEYWORDS - beta-Lactams; Carbamazepine; Clindamycin; Drug Eruptions; Drug Hypersensitivity; Hypersensitivity, Delayed/ chemically induced; Patch Tests.

\section{INTRODUÇÃO}

As reações adversas medicamentosas cutâneas (RAMc) representam $20-30 \%$ dos efeitos adversos a medicamentos e os antibióticos e os anticonvulsivantes estão frequentemente implicados em reações retardadas (não imediatas) mediadas por células $T$, nomeadamente exantema maculopapular, pustulose exantemática aguda generalizada (PEAG), síndrome de hipersensibilidade a fármacos (DRESS) e síndrome de Stevens-Johnson/necrólise epidérmica tóxica. ${ }^{1-3}$

Os testes epicutâneos têm-se revelado úteis no estudo destas RAMc retardadas, tanto a antibióticos, como a anticonvulsivantes, com alta especificidade mas uma sensibilidade variável em função do fármaco imputável e do tipo de erupção medicamentosa. ${ }^{4-7}$

De acordo com as recomendações atuais para o estudo das RAMc retardadas, os testes epicutâneos devem ser realizados entre 3-6 semanas até 3-6 meses após a resolução da erupção cutânea. ${ }^{8,9}$ No entanto, testes epicutâneos positivos foram pontualmente observados, pela primeira, vez vários anos ou décadas após a erupção cutânea inicial. ${ }^{10}$ De forma semelhante, as células mononucleares periféricas podem manter a capacidade para ser especificamente estimuladas por betalactâmicos ou anticonvulsivantes, por longos períodos após a resolução do quadro cutâneo, mesmo na ausência de estimulação adicional, o que sugere um efeito memória. ${ }^{6,11}$ Considera-se assim, à semeIhança da alergia de contacto a pequenas moléculas, que nas RAMc retardadas a antibióticos e a anticonvulsivantes a hipersensibilidade mediada por células T é persistente, eventualmente para toda a vida. ${ }^{12,13}$

$\mathrm{Na}$ dermatite de contacto alérgica a reprodutibilidade dos testes epicutâneos positivos varia entre 66 e $71 \%$ e não parece ser afetada pelo intervalo decorrido entre os testes. ${ }^{13-15} \mathrm{Na}$ dermatite de contacto alérgica ocupacional às penicilinas foi demonstrado que os testes epicutâneos, quando repetidos após longo prazo, tendem a manter-se positivos, ainda que com oscilações na intensidade. ${ }^{16}$

No contexto das RAMc retardadas aos antibióticos e a anticonvulsivantes é escassa a evidência a favor da reprodutibilidade a longo prazo dos testes epicutâneos positivos e, consequentemente, da persistência da hipersensibilidade para toda a vida. Também são escassos os estudos de repetição da provocação oral ao fim de muitos anos.

Esta falta de evidência torna difícil a recomendação de evicção para toda a vida de medicamentos tantas vezes importantes para os doentes.

\section{OBJETIVO}

Avaliar, a longo prazo, a persistência das reações positivas a medicamentos que frequentemente causam testes epicutâneos positivos, nomeadamente betalactâmicos, clindamicina e carbamazepina, no contexto de RAMc retardadas.

\section{MATERIAL E MÉTODOS}

Inicialmente foram revistos os processos clínicos e fichas alergológicas de todos os doentes com antecedentes de RAMc retardada a antibióticos betalactâmicos, clindamicina e carbamazepina e testes epicutâneos positivos (pelo menos 1+, com eritema e infiltração em toda a área do teste epicutâneo), realizados na Unidade de Alergologia de Contacto do nosso Serviço de Dermatologia, entre os anos 2000 e 2015. A restrição dos antibióticos estudados aos betalactâmicos e à clindamicina deveu-se ao facto de estes serem os que mais frequentemente se associaram a testes positivos no nosso serviço nos últimos anos. ${ }^{4} \mathrm{O}$ mesmo é válido para a carbamazepina, no caso dos anticonvulsivantes. ${ }^{7}$

Estes doentes foram posteriormente entrevistados telefonicamente acerca de nova exposição aos fármacos imputáveis com possível RAMc (após os testes epicutâneos iniciais). Naqueles em que tal exposição não ocorreu foi proposta a repetição dos testes epicutâneos, pelo menos 2 anos após a realização dos primeiros testes.

Adicionalmente, os doentes com reações positivas em testes epicutâneos anteriores ao ano 2000, que por algum motivo foram novamente referenciados à mesma Unidade de Alergologia de Contacto durante o período do estudo, foram igualmente convidados a realizar novos testes, desde que não houvesse história de nova exposição ao fármaco imputável.

O estudo enquadrou-se dentro de um estudo mais alargado de caracterização dos testes epicutâneos em RAMc aprovado pela Comissão de ética da Faculdade de Medicina da Universidade de Coimbra e os doentes participaram voluntariamente, após assinatura do consentimento informado para a realização dos testes epicutâneos.

Foram identificados 64 doentes elegíveis e 23 aceitaram participar no estudo (taxa de resposta de 35,9\%). Tanto os testes epicutâneos iniciais como os testes repetidos foram realizados de acordo com as recomendações da European Society of Contact Dermatitis (ESCD) e da European Network on Drug Allergy/European Academy of Allergy and Clinical Immunology. $8,9,17$ Os testes foram aplicados na região dorsal superior durante 2 dias, utilizando Finn Chambers em Scanpor tape ${ }^{\circledR}$ (Epitest Ltd Oy, Tuusula, Finlândia) ou IQ-Ultra Chambers ${ }^{T M}$ (Chemotechnique Diagnostics, Vellinge, Suécia), de acordo 
com o material disponível. Foram efetuadas duas leituras, uma ao $2^{\circ}$ dia (D2) e outra em D3 ou D4, com uma leitura adicional em D7, sempre que os resultados iniciais foram negativos. Foram utilizados os critérios de leitura recomendados pela ESCD para avaliar as reações dos testes epicutâneos no estudo da alergia de contacto. ${ }^{8}$

Em todos os participantes com testes iniciais positivos a antibióticos foi testada uma série de pelo menos 32 antibióticos sistémicos, incluindo os 16 fornecidos pela Chemotechnique Diagnostics. Os outros antibióticos não comercializados em teste epicutâneo foram preparados localmente, a partir dos fármacos comercializados em cápsula, comprimido ou pó para solução injetável, manipulados em vaselina esterilizada por forma a obter concentrações finais do antibiótico na preparação de 10\% (Tabela 1). Nos casos com RAMc à carbamazepina foram testadas três concentrações deste fármaco, a $1 \%$, e 10\% em vaselina (Chemotechnique Diagnostics) e a $20 \%$ em vaselina preparado localmente a partir do pó de carbamazepina (Sigma-Aldrich, Portugal)

As reações dos testes epicutâneos foram avaliadas quanto: 1) à persistência da positividade a longo prazo nos novos testes epicutâneos (considerando-se a reação reprodutível); 2) à variação da intensidade ao longo do tempo (comparando-se intensidade máxima obtida com a mesma concentração do alergénio nos dois testes); 3) à influência da idade, género e tempo entre testes na positividade a longo prazo dos testes.

\section{ANÁLISE ESTATÍSTICA}

Os dados foram analisados com o software SPSS (Version 21.0. Armonk, NY: IBM Corp., USA). Foi utilizado o teste exato de qui-quadrado para estudar a influência do género na variação da intensidade das reações dos novos testes. $\bigcirc$ teste de Kruskal-Wallis permitiu avaliar o efeito do intervalo de tempo entre os testes e da idade à data da sua realização, na variação da intensidade das reações. Um valor de $p<0,05$ foi considerado significativo.

\section{RESULTADOS \\ I - Caraterização dos casos com testes inicialmen- te positivos}

Os 23 doentes incluídos, 10 homens e 13 mulheres, apresentavam à data dos primeiros testes uma idade mediana de

Tabela 1 - Alergénios incluídos na nossa série de antibióticos para testes epicutâneos.

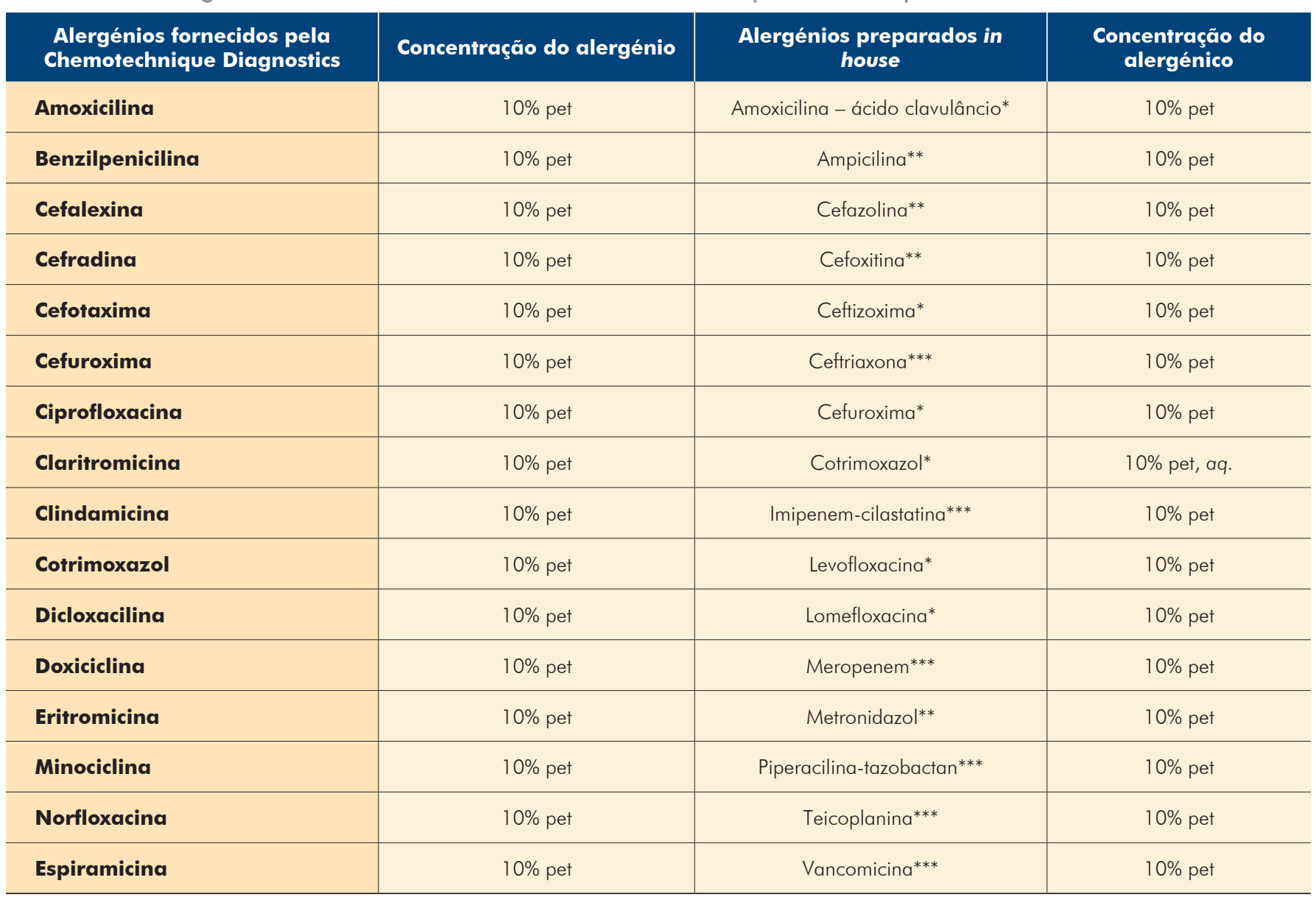

*alergénio preparado a partir de comprimido ou cápsula cedido pela farmácia hospitalar; ** alergénio preparado a partir do fármaco puro cedido por companhia farmacêutica; ${ }^{* * *}$ alergénio preparado a partir de pó para injeção intravenosa cedido pela farmácia hospitalar; pet - vaselina; aq. - água. 


\section{GPDEC}

Tabela 2 - Caraterização dos casos com reações inicialmente positivas, que foram submetidos a novos testes epicutâneos.

\begin{tabular}{|c|c|c|c|c|c|c|c|c|c|}
\hline $\begin{array}{l}\text { Caso / } \\
\text { género }\end{array}$ & $\begin{array}{l}\text { Idade ao } \\
1^{\circ} \mathrm{TE}\end{array}$ & $\begin{array}{l}\text { Idade ao } \\
\text { novo TE }\end{array}$ & $\begin{array}{l}\text { Intervalo } \\
\text { entre TE } \\
\text { (anos) }\end{array}$ & RAMC & $\begin{array}{l}\text { Fármaco(s) } \\
\text { imputável }\end{array}$ & $\begin{array}{r}\text { Reaçóes no } \\
\text { Leituras em D2 }\end{array}$ & $\begin{array}{l}\text { TE } \\
\text { D3/D4 }\end{array}$ & $\begin{array}{r}\text { Reaçōes no } \\
\text { Leituras em D }\end{array}$ & $\begin{array}{l}\circ \mathrm{TE} \\
\text { 33/D4 }\end{array}$ \\
\hline $1-M$ & 13 & 41 & 30,7 & DRESS & $\begin{array}{l}\text { AMX } \\
\text { Carbamazepina }\end{array}$ & $\begin{array}{l}\text { AMX** } \\
\text { AMP } \\
\text { Benzilpenicilina } \\
\text { Carbamazepina }\end{array}$ & $\begin{array}{l}+++ \\
+++ \\
+++ \\
+++\end{array}$ & $\begin{array}{l}\text { AMX } \\
\text { AMP } \\
\text { Benzilpenicilina } \\
\text { Carbamazepina }\end{array}$ & $\begin{array}{l}+++ \\
++ \\
+++ \\
+\end{array}$ \\
\hline $2-F$ & 31 & 46 & 14,7 & EMP & AMX* & $\begin{array}{l}\text { AMX } \\
\text { AMP }\end{array}$ & $\begin{array}{l}++ \\
++\end{array}$ & $\begin{array}{l}\text { AMX } \\
\text { AMP }\end{array}$ & $\begin{array}{l}++ \\
+\end{array}$ \\
\hline $3-M$ & 37 & 49 & 12,5 & EMP & AMX & $\begin{array}{l}\text { AMX } \\
\text { AMP }\end{array}$ & $\begin{array}{l}++ \\
++ \\
\end{array}$ & $\begin{array}{l}\text { AMX } \\
\text { AMP }\end{array}$ & $\begin{array}{l}++ \\
+++\end{array}$ \\
\hline $4-F$ & 64 & 76 & 11,5 & EMP & Clindamicina * & Clindamicina & ++ & Clindamicina & ++ \\
\hline $5-F$ & 51 & 60 & 10,7 & EMP & Flucloxacilina & $\begin{array}{l}\text { Flucloxacilina } \\
\text { Dicloxacilina } \\
\text { Benzilpenicilina }\end{array}$ & $\begin{array}{l}+++ \\
+++ \\
+++\end{array}$ & $\begin{array}{l}\text { Flucloxacilina } \\
\text { Dicloxacilina } \\
\text { Benzilpenicilina }\end{array}$ & $\begin{array}{l}++ \\
++ \\
+\end{array}$ \\
\hline $6-F$ & 33 & 42 & 8,5 & EMP & Clindamicina & Clindamicina & ++ & Clindamicina & $\begin{array}{l}\mathrm{Neg}- \\
\mathrm{NR}\end{array}$ \\
\hline $7-F$ & 67 & 75 & 7,9 & EMP & Clindamicina* & Clindamicina & ++ & Clindamicina & + \\
\hline $8-F$ & 72 & 79 & 7,4 & EMP & AMX & $\begin{array}{l}\text { AMX } \\
\text { AMP }\end{array}$ & $\begin{array}{l}+++ \\
+++\end{array}$ & $\begin{array}{l}\text { AMX } \\
\text { AMP }\end{array}$ & $\begin{array}{l}+ \\
+\end{array}$ \\
\hline $9-F$ & 55 & 62 & 7,0 & EMP & Clindamicina & Clindamicina & + & Clindamicina & + \\
\hline $10-M$ & 36 & 43 & 6,5 & PEAG & $\begin{array}{l}\text { AMX } \\
\text { Flucloxacilina }\end{array}$ & $\begin{array}{l}\text { AMX } \\
\text { AMP } \\
\text { Flucloxacilina } \\
\text { Dicloxacilina } \\
\text { Benzilpenicilina } \\
\text { Piperacilina }\end{array}$ & $\begin{array}{l}+ \\
+ \\
+++ \\
++ \\
++ \\
++\end{array}$ & $\begin{array}{l}\text { AMX } \\
\text { AMP } \\
\text { Flucloxacilina } \\
\text { Dicloxacilina } \\
\text { Benzilpenicilina } \\
\text { Piperacilina }\end{array}$ & $\begin{array}{l}++ \\
+ \\
+++ \\
++ \\
++ \\
++\end{array}$ \\
\hline $11-M$ & 56 & 62 & 5,8 & EMP & Espiramicina & Clindamicina & + & Clindamicina & ++ \\
\hline $12-M$ & 70 & 85 & 4,9 & EMP & Flucloxacilina & $\begin{array}{l}\text { Dicloxacilina } \\
\text { Flucloxacilina }\end{array}$ & $\begin{array}{l}+ \\
+ \\
+\end{array}$ & $\begin{array}{l}\text { Dicloxacilina } \\
\text { Flucloxacilina }\end{array}$ & $\begin{array}{l}+ \\
+ \\
+\end{array}$ \\
\hline $13-F$ & 39 & 45 & 5,2 & DRESS & Carbamazepina & Carbamazepina & + & Carbamazepina & + \\
\hline $14-F$ & 40 & 45 & 4,8 & DRESS & Carbamazepina & Carbamazepina & ++ & Carbamazepina & ++ \\
\hline $15-M$ & 51 & 54 & 3,7 & DRESS & $\begin{array}{l}\text { AMX } \\
\text { Carbamazepina }\end{array}$ & $\begin{array}{l}\text { AMX } \\
\text { AMP } \\
\text { Carbamazepina }\end{array}$ & $\begin{array}{l}+ \\
++ \\
+\end{array}$ & $\begin{array}{l}\text { AMX } \\
\text { AMP } \\
\text { Carbamazepina }\end{array}$ & $\begin{array}{l}\text { Neg- } \\
\text { NR } \\
\text { Neg- } \\
\text { NR } \\
+ \\
\end{array}$ \\
\hline $16-F$ & 35 & 39 & 3,4 & EMP & Clindamicina & Clindamicina & + & Clindamicina & $\begin{array}{l}\text { Neg- } \\
\text { NR }\end{array}$ \\
\hline $17-F$ & 53 & 55 & 2,6 & EMP & AMX & $\begin{array}{l}\text { AMX } \\
\text { AMP }\end{array}$ & $\begin{array}{l}++ \\
++\end{array}$ & $\begin{array}{l}\text { AMX } \\
\text { AMP }\end{array}$ & $\begin{array}{l}+ \\
+\end{array}$ \\
\hline 18-M & 26 & 28 & 2,6 & EMP & AMX & $\begin{array}{l}\text { AMX } \\
\text { AMP }\end{array}$ & $\begin{array}{l}++ \\
++\end{array}$ & $\begin{array}{l}\text { AMX } \\
\text { AMP }\end{array}$ & $\begin{array}{l}++ \\
+++\end{array}$ \\
\hline $19-F$ & 60 & 62 & 2,3 & EMP & AMX & $\begin{array}{l}\text { AMX } \\
\text { AMP }\end{array}$ & $\begin{array}{l}++ \\
++\end{array}$ & $\begin{array}{l}\text { AMX } \\
\text { AMP }\end{array}$ & $\begin{array}{l}++ \\
++\end{array}$ \\
\hline 20- M & 64 & 65 & 2,2 & EMP & Cefoxitina & Cefoxitina & + & Cefoxitina & + \\
\hline $21-M$ & 60 & 62 & 2,0 & EMP & Clindamicina* & Clindamicina & ++ & Clindamicina & ++ \\
\hline $22-F$ & 26 & 28 & 2,0 & EMP & Flucloxacilina & $\begin{array}{l}\text { Flucloxacilina } \\
\text { Dicloxacilina }\end{array}$ & $\begin{array}{l}+++ \\
+++\end{array}$ & $\begin{array}{l}\text { Flucloxacilina } \\
\text { Dicloxacilina }\end{array}$ & $\begin{array}{l}+++ \\
+++\end{array}$ \\
\hline $23-M$ & 16 & 18 & 2,0 & DRESS & $\begin{array}{l}\text { AMX } \\
\text { Carbamazepina }\end{array}$ & $\begin{array}{l}\text { AMX } \\
\text { Carbamazepina }\end{array}$ & $\begin{array}{l}+ \\
+ \\
+\end{array}$ & $\begin{array}{l}\text { AMX } \\
\text { Carbamazepina }\end{array}$ & $\begin{array}{l}+ \\
++ \\
\end{array}$ \\
\hline
\end{tabular}

AMX - amoxicilina; AMP - ampicillina; DRESS - drug reaction with eosinophilia and systemic symptoms; EMP - exantema maculopapular; F - feminino; M - masculino; Neg-NR - reação negativa, não reprodutível; PEAG - pustulose exantemática aguda generalizada; TE - testes epicutâneos 
51,0 anos (min. 13 - máx. 72 anos) e 17 destes tinham história de exantema maculopapular (EMP), cinco de drug reaction with eosinophilia and systemic symptoms (DRESS) e um de pustulose exantemática aguda generalizada (PEAG).

Nesta população foram inicialmente observadas 44 reações positivas, 27 ao fármaco(s) imputável(is) e 17 reações positivas, explicáveis por possíveis reações cruzadas. Por ordem decrescente de frequência, os antibióticos imputáveis mais prevalentes foram a amoxicilina (em nove casos), clindamicina (em sete), flucloxacilina (em quatro) e cefoxitina (em um). As reações cruzadas positivas nos primeiros testes foram com a ampicilina (nove casos), dicloxacilina (quatro casos), benzilpenicilina (três casos) e piperacilina (um caso). Foram ainda observadas cinco reações positivas à carbamazepina (todas no contexto de DRESS), sendo que em dois casos havia simultaneamente reatividade à amoxicilina (fármaco igualmente imputável).

\section{II - Reatividade a longo prazo das reações posi- tivas}

Os novos testes epicutâneos foram reprodutíveis (em relação ao número de reações positivas) em 20/23 (87,0\%) doentes, nove homens e 11 mulheres, após um intervalo de tempo mediano total de 6,5 anos (min. 2,0 - máx. 30,7).

Apenas quatro (10,3\%) das 39 reações inicialmente positivas aos antibióticos deixaram de se observar nos novos testes, enquanto $35(89,7 \%)$ se mantiveram positivas (Tabela 2$)$.

Em relação aos betalactâmicos, 9/10 testes com amoxicilina, 4/4 com flucloxacilina e 1/1 com cefoxitina continuaram positivos, após um tempo mediano de 6,5, 5,7 e 2,2 anos, respetivamente. No caso das reações cruzadas (com a amoxicilina), 8/9 testes com ampicilina, $3 / 3$ com benzilpenicilina e $1 / 1$ com piperacilina persistiram reativos, após um tempo mediano de 6,6, 10,7 e 6,5 anos, respetivamente. Os quatro doentes com testes positivos à flucloxacilina também reagiram para dicloxacilina.

Todas as reações com betalactâmicos que ocorreram no contexto de EMP e de PEAG mantiveram-se positivas nos novos testes.

Dos sete testes positivos à clindamicina, todos em casos de EMP, $5(71,4 \%)$ permaneceram positivos, após uma mediana de 7,0 anos.

Nos três casos de DRESS em que a carbamazepina foi o único fármaco imputável, todos os novos testes se mantiveram positivos, após 4,0 anos. Noutros dois casos, o DRESS foi imputável à amoxicilina e carbamazepina e estes dois fármacos foram positivos no teste inicial. Num caso (\# 1) a repetição dos testes após 30 anos mostrou um teste positivo à amoxicilina e negativo à carbamazepina a 1 e 10\% em vaselina (tendo sido possível uma única leitura em D2), mas oito meses mais tarde os testes com carbamazepina a 10 e $20 \%$ em vaselina foram positivos (em D3), assim como com a amoxicilina. $O$ outro caso (\#15) perdeu reatividade à amoxicilina (após 3,7 anos), mas manteve a reatividade à carbamazepina. $\bigcirc$ intervalo de tempo mediano entre testes à carbamazepina foi de 12,0 anos.
A maioria das reações positivas nos novos testes epicutâneos foi observada em D2 e nenhuma ocorreu após D4. Em relação à intensidade, as reações foram sobretudo $1+$ $(16 / 40)$ ou $2+(17 / 40)$, sendo apenas $7 / 40$ as reações $3+$.

Não ocorreu nenhuma exacerbação da RAMc inicial durante os testes epicutâneos. Não ocorreram reações falsamente positivas com os outros fármacos incluídos na nossa série.

\section{III - Variação da intensidade das reações a longo prazo}

Das 22 reações com os antibióticos imputáveis, três $(18,2 \%)$ tornaram-se negativas nos novos testes, enquanto 19 $(86,4 \%)$ se mantiveram positivas, 14 das quais $(73,7 \%) \mathrm{com} \mathrm{a}$ mesma intensidade.

Em relação às reações cruzadas, 16/17 (94,1\%) permaneceram positivas, mas a proporção cuja intensidade se manteve inalterada foi inferior àquela observada com os fármacos imputáveis $(8 / 16,50 \%)$.

Com respeito à carbamazepina, a intensidade dos cinco novos testes manteve-se igual em três (60\%) ocasiões, diminuiu em uma ocasião e aumentou noutra, tendo sido documentada numa realização dos testes uma resposta negativa à carbamazepina quando testada apenas até $10 \%$ vas.

No global, 62,5\% das reações positivas nos novos testes mantiveram a mesma intensidade dos testes iniciais, enquanto $12,5 \%$ aumentaram e $27,5 \%$ diminuíram de intensidade.

Comparando as reações cuja intensidade se manteve inalterada com aquelas em que a intensidade variou (aumentou, ou diminuiu), não se observou diferença estatisticamente significativa no intervalo de tempo entre os testes, idade à data da sua realização, ou género $(p>0,05)$. Também não se encontrou diferença estatisticamente significativa nestas variáveis quando as reações inicialmente positivas foram comparadas com aquelas que se tornaram negativas.

\section{DISCUSSÃO}

Com este trabalho foi possível demonstrar que $87,0 \%$ dos doentes com testes epicutâneos inicialmente positivos, no contexto de RAMc retardadas a antibióticos e anticonvulsivantes, mantiveram a hipersensibilidade retardada por vários anos. Adicionalmente, 90, 1\% das reações iniciais continuaram positivas, na maioria dos casos com a mesma intensidade. Foi ainda possível demonstrar que a reprodutibilidade dos testes epicutâneos não foi afetada pelo tempo decorrido entre testes, nem pela idade ou género dos doentes.

Estes dados contrariam aquilo que está descrito para as RAMc imediatas. Em estudos prévios que avaliaram a evolução temporal da hipersensibilidade imediata a antibióticos, por meio de doseamento de lgE específicas e de testes da picada, verificou-se que na ausência de exposição adicional aos fármacos, esta tende a desaparecer. ${ }^{18,19}$ Estima-se que, 5 anos após a erupção cutânea, em metade dos casos com alergia à penicilina mediada por lgE não seja possível documentar esta hipersensibilidade, pelo teste da picada ou $\lg \mathrm{E}$ sérica específica, e que esta percentagem aumente para $80 \%$ aos 10 anos. ${ }^{20,21}$ 


\section{GPDEC}

Tabela 3 - Variação da intensidade das reações dos testes epicutâneos.

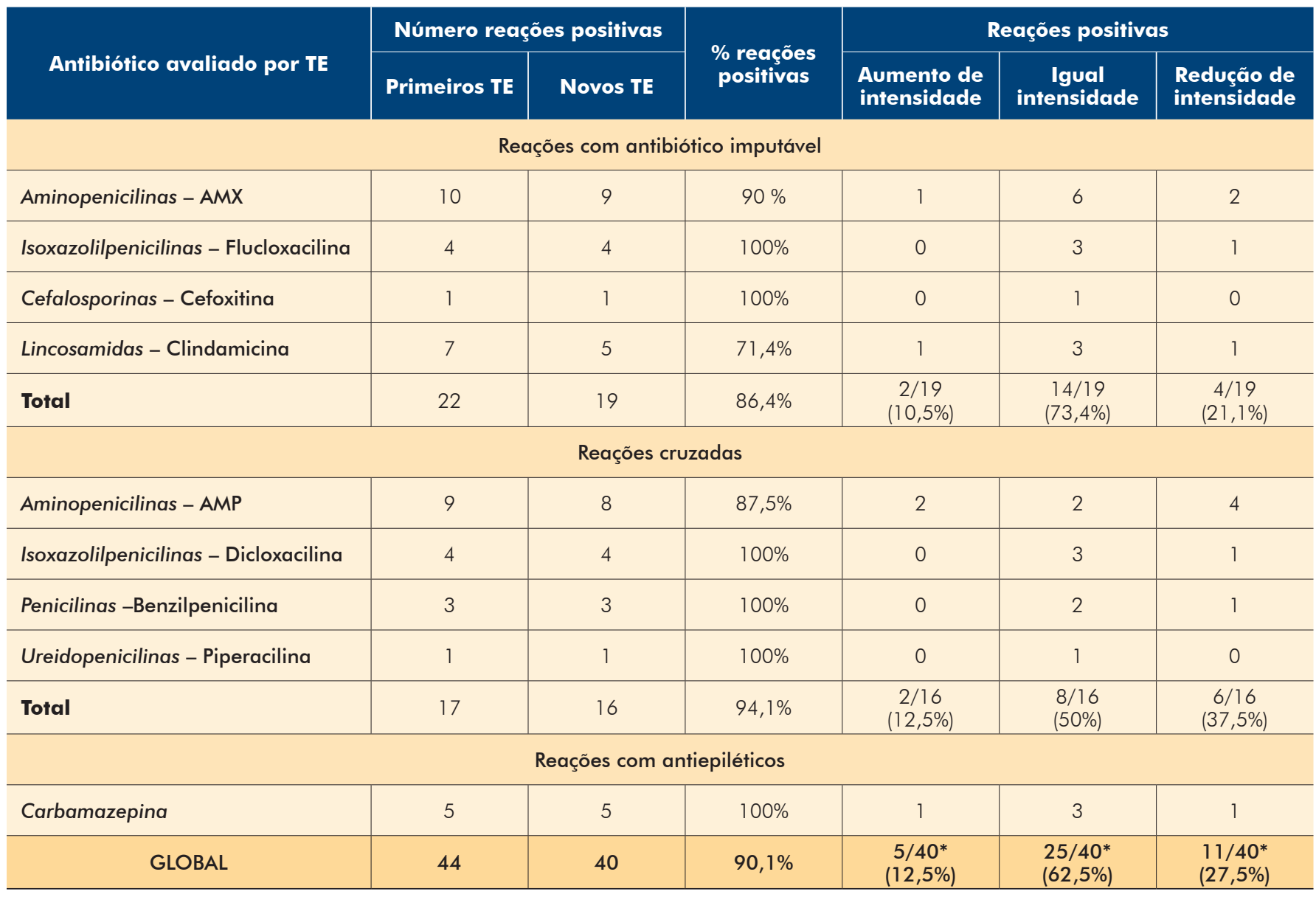

AMX - amoxicilina; AMP - ampicilina; TE - testes epicutâneos; *sem diferença estatisticamente significativa em relação ao género ( $p=0,6$, teste qui-quadrado), idade ( $p=0,9$, teste Kruskal-Wallis) ou intervalo entre testes ( $p=0,1$, teste Kruskal-Wallis)

No contexto das RAMc a antibióticos poucos estudos procuraram avaliar, por meio de testes epicutâneos, a evolução temporal da hipersensibilidade retardada.

Num estudo de 1981, Bruynzeel e van Ketel testaram repetidamente 15 pacientes com hipersensibilidade retardada à amoxicilina, ampicilina ou feneticilina. ${ }^{22}$ Os testes foram realizados sequencialmente às 10, 16, 64 e 100 semanas, após a erupção medicamentosa inicial, com os fármacos imputáveis, além de penicilina $G$, cloxacilina, cefradina e cefalotina. Em 12 dos 15 (80\%) doentes os testes permaneceram positivos. Em oito casos o número de reações positivas aumentou, ou seja, surgiram novos testes positivos presumivelmente por reações cruzadas, e em sete destes houve um aumento da intensidade das reações. Em cinco casos o número de reações diminuiu; em sete doentes as reações tornaram-se menos intensas. Ainda assim, não foi discriminado o padrão de toxidermia, nem foi feita distinção do padrão de reatividade em função de fármaco imputável.

Uma década mais tarde, Romano et al mostraram que em 23 doentes com episódio prévio de urticária, angioedema ou EMP (mas sem ser discriminada a proporção de casos de cada reação) e testes epicutâneos positivos à amoxicilina ou ampicilina, a sua repetição, pelo menos um ano mais tarde, levou a resultados positivos em todos os casos. Em 16/23 $(69,5 \%)$ destes a intensidade das reações manteve-se inalterada. ${ }^{23}$ De forma semelhante, na nossa série, das 19 reações positivas com fármaco imputável $14(73,4 \%)$ mantiveram a mesma intensidade, sendo esta proporção inferior nas reações cruzadas (50\%).

Com este trabalho foi possível documentar que no contexto de EMP e de AGEP a reatividade a longo prazo com os betalactâmicos foi a regra, mantendo-se a totalidade dos testes positivos.

Uma alta proporção de testes positivos foi igualmente observada com a clindamicina $(71,4 \%)$, um fármaco para 0 qual a sensibilidade diagnóstica de RAMc retardadas dos testes epicutâneos varia entre 19-30\%. ${ }^{24,25}$

Em relação à carbamazepina demonstrámos que nos cinco casos de DRESS, a hipersensibilidade retardada ao anticonvulsivante se manteve positiva, após um tempo mediano de 12,0 anos. A reprodutibilidade destes testes tem sido apenas descrita de forma esporádica, em alguns relatos de caso, 
sendo mais frequente a descrição de testes positivos pela primeira vez, vários anos após um quadro bem documentado de DRESS à carbamazepina. ${ }^{26,27}$

Importa, contudo, distinguir os casos de DRESS em que o fármaco imputável era apenas a carbamazepina, daqueles em qua houve simultaneamente exposição à amoxicilina, provavelmente para tratamento da sintomatologia prodrómica do DRESS, (ou durante o estado de imunossupressão que carateriza o início desta toxidermia). Em alguns casos publicados na última década tem sido sugerido que durante um episódio de DRESS à carbamazepina a exposição a outros fármacos não aparentados, tais como amoxicilina, amitriptilina ou paracetamol pode levar a cossensibilização, com novo surto de DRESS quando há exposição a estes fármacos, na ausência da carbamazepina. ${ }^{26,27}$ Especula-se que a estimulação massiva não específica do sistema imune, que sucede o estado de imunossupressão inicial durante um episódio de DRESS à carbamazepina (e que pode facilitar a expressão de moléculas coestimulatórias e citocinas pró-inflamatórias), facilite a apresentação antigénica e reduza a tolerância a fármacos imunogénicos como a amoxicilina, com eventual desenvolvimento de cossensibilização. ${ }^{27}$

Nós verificamos, no entanto, que dos dois casos de DRESS com testes positivos à carbamazepina e amoxicilina (além da ampicilina - reação cruzada), em apenas um esta cossensibilização persistiu, após 3 décadas, enquanto noutro se perdeu em menos de 4 anos (mesmo após testes epicutâneos inicialmente positivos). Seria interessante, avaliar por testes in vitro (testes de transformação linfocitária, ELISpot) ou in vivo (testes intradérmicos, ou mesmo provocação oral) se esta perda de reatividade é inerente aos testes epicutâneos, ou transversal a todos testes.

Uma implicação prática decorrente deste estudo prende-se com a redefinição do timing para a realização de testes epicutâneos e, consequentemente, até que ponto podem ser utilizados no diagnóstico retrospetivo de uma RAMc retardada a antibióticos e anticonvulsivantes.

Sabe-se que a realização ocasional de testes epicutâneos tem pouco ou nenhum potencial para desencadear sensibilização de novo e que a ocorrência de reações falsamente positivas é um fenómeno raro. ${ }^{28}$ Assim sendo, é possível que para maioria destes doentes, se os testes epicutâneos tivessem sido realizados pela primeira vez 6,5 anos após o episódio de RAMc retardada, (o tempo mediano para repetição dos testes neste estudo) a taxa de positividade das reações seria superior a $90 \%$, revelando-se os testes úteis no estabelecimento de nexo de causalidade entre o fármaco imputável e a erupção medicamentosa. Com base nesta inferência, talvez não haja indicação para "limitar" o intervalo para a realização dos testes nas RAMc retardadas aos 3-6 meses, e mesmo que o limite tenha sido ultrapassado, os testes mantêm a sua utilidade, sobretudo quando se pretender clarificar o diagnóstico de uma toxidermia antiga.

Este estudo apresenta algumas limitações metodológicas. $O$ gold standard para avaliação das RAMc retardadas é considerado a provocação oral. No entanto, sendo os testes epicutâneos uma técnica segura e específica consideramo-la válida para este estudo, permitindo evitar os riscos potenciais inerentes à prova de provocação oral ou à reintrodução clínica acidental do fármaco. Com alguns dos testes a serem realizados antes do ano 2001, é possível que a interpretação da intensidade das reações não tenha sido exatamente a mesma daquela dos testes atuais, não permitindo uma comparação de intensidade totalmente fidedigna.

\section{CONCLUSÃO}

No contexto de RAMc retardadas a medicamentos que frequentemente causam testes epicutâneos positivos, a maioria das reações positivas com antibióticos - betalactâmicos, clindamicina - e a totalidade das reações positivas com carbamazepina persistiram positivas por vários anos.

Estes dados reforçam a recomendação para evicção, para toda a vida, dos fármacos altamente imputáveis e, sobretudo, os confirmados como agente etiológico de RAMc retardada, mas também enfatizam o papel dos testes epicutâneos no diagnóstico retrospetivo da toxidermia, mesmo quando esta ocorreu vários anos antes. Permitem ainda sugerir o alargamento do intervalo de tempo proposto nas recomendações europeias para realização dos testes epicutâneos, sendo aceitável ultrapassar os 6 meses após a resolução da toxidermia.

Conflitos de interesse: Os autores declaram não possuir conflitos de interesse.

Suporte financeiro: $O$ presente trabalho não foi suportado por nenhum subsídio ou bolsa.

Confidencialidade dos dados: Os autores declaram ter seguido os protocolos do seu centro de trabalho acerca da publicação dos dados de doentes.

Protecção de pessoas e animais: Os autores declaram que os procedimentos seguidos estavam de acordo com os regulamentos estabelecidos pelos responsáveis da Comissão de Investigação Clínica e Ética e de acordo com a Declaração de Helsínquia da Associação Médica Mundial

Conflicts of interest: The authors have no conflicts of interest to declare.

Financing Support: This work has not received any contribution, grant or scholarship.

Confidentiality of data: The authors declare that they have followed the protocols of their work center on the publication of data from patients.

Protection of human and animal subjects: The authors declare that the procedures followed were in accordance with the regulations of the relevant clinical research ethics committee and with those of the Code of Ethics of the World Medical Association (Declaration of Helsinki).

\section{REFERÊNCIAS}

1. Svensson CK, Cowen EW, Gaspari AA. Cutaneous drug reactions. Pharmacol Rev. 2001;53:357-79.

2. Mockenhaupt M. Epidemiology of cutaneous adverse drug reactions. Chem Immunol Allergy. 2012;97:1-17.

3. Gonçalo M, Canelas MM, Cardoso JC. Mecanismos fisiopatológicos envolvidos nas principais reacções de 
hipersensibilidade medicamentosa. Trab Soc Port Derm Vener 2009;67:385-407.

4. Pinho A, Coutinho I, Gameiro A, Gouveia M, Goncalo M. Patch testing - a valuable tool for investigating non-immediate cutaneous adverse drug reactions to antibiotics. J Eur Acad Dermatol Venereol. 2017. 31:280-7.

5. Barbaud A, Collet E, Milpied B, Assier H, Staumont D, Avenel-Audran $M$, et al. A multicentre study to determine the value and safety of drug patch tests for the three main classes of severe cutaneous adverse drug reactions. $\mathrm{Br} \mathrm{J}$ Dermatol. 2013;168:555-62.

6. Pichler WJ, Tilch J. The lymphocyte transformation test in the diagnosis of drug hypersensitivity. Allergy. 2004;59:80920.

7. Santiago F, Gonçalo M, Vieira R, Coelho S, Figueiredo A. Epicutaneous patch testing in drug hypersensitivity syndrome (DRESS). Contact Dermatitis. 2010;62:47-53.

8. Johansen JD, Aalto-Korte K, Agner T, Andersen KE, Bircher $A$, Bruze $M$, et al. European Society of Contact Dermatitis guideline for diagnostic patch testing - recommendations on best practice. Contact Dermatitis. 2015;73:195-221.

9. Brockow K, Garvey LH, Aberer W, Atanaskovic-Markovic $M$, Barbaud $A$, Bilo $M B$, et al. Skin test concentrations for systemically administered drugs - an ENDA/EAACI Drug Allergy Interest Group position paper. Allergy. 2013;68:702-12.

10. Torres MJ, Sánchez-Sabaté E, Álvarez J, Mayorga C, Fernández J, Padial A, et al. Skin test evaluation in nonimmediate allergic reactions to penicillins. Allergy. 2004;59:219-24.

11. Fu M, Gao Y, Pan Y, Li W, Liao W, Wang G, et al. Recovered patients with Stevens-Johson syndrome and toxic epidermal necrolysis maintain long-lived IFN- $\gamma$ and sFasL memory response. PLoS One. 2012;7:e45516.

12. Romano A, Blanca M, Torres MJ, Bircher A, Aberer W, Brockow K, et al. Diagnosis of nonimmediate reactions to beta-lactam antibiotics. Allergy. 2004;59:1 153-60.

13. Jensen CD, Andersen KE. Course of contact allergy in consecutive eczema patients patch tested with TRUE Test ${ }^{\mathrm{TM}}$ panels 1 and 2 at least twice over a 12-year period. Contact Dermatitis. 2005;52:242-6.

14. Schaeffer AC, Andersen KE, Bindslev-Jensen C, Mortz CG. The reproducibility of nickel, cobalt and chromate sensitization in patients tested at least twice in the period 1992-2014 with TRUE Test(R). Contact Dermatitis. 2016;75:111-3.

15. Nielsen NH, Linneberg A, Menné T, Madsen F, Frølund L,
Dirksen A, et al. Persistence of contact allergy among Danish adults: an 8-year follow-up study. Contact Dermatitis. $2001 ; 45: 350-3$.

16. Møller NE, Jeppesen K. Patch testing with semisynthetic penicillins. Contact Dermatitis. 1987;16:227-8.

17. Brockow K, Romano A, Blanca M, Ring J, Pichler W, Demoly P. General considerations for skin test procedures in the diagnosis of drug hypersensitivity. Allergy. 2002;57:4551.

18. Zvonar RK. Cross-allergy among the B-lactam antibiotic agents: a review of the risks. Can J Hospl Pharm. 2009;58.

19. Joint Task Force on Practice Parameters; American Academy of Allergy, Asthma and Immunology.; American College of Allergy, Asthma and Immunology.; Joint Council of Allergy, Asthma and Immunology. Drug allergy: an updated practice parameter. Ann Allergy Asthma Immunol. 2010;105:259-73.

20. Blanca M, Torres MJ, García JJ, Romano A, Mayorga C, de Ramon $E$, et al. Natural evolution of skin test sensitivity in patients allergic to $\beta$-lactam antibiotics. J Allergy Clinl Immunol. 1999;103:918-24.

21. Sullivan TJ, Wedner HJ, Shatz GS, Yecies LD, Parker CW. Skin testing to detect penicillin allergy. J Allergy Clin Immunol. 1981;68:171-80.

22. Bruynzeel DP, Van Ketel WG. Repeated patch testing in penicillin allergy. Br J Dermatol. 1981;104:157-9.

23. Romano A, Fonso MD, Pietrantonio F, Pocobelli D, Giannarini L, Del Bono A, et al. Repeated patch testing in delayed hypersensitivity to beta-lactam antibiotics. Contact Dermatitis 1993;28:190.

24. Lammintausta K, Kortekangas-Savolainen O. The usefulness of skin tests to prove drug hypersensitivity. Br J Dermatol. 2005; 152:968-74.

25. Pereira N, Canelas MM, Santiago F, Brites MM, Gonçalo $M$. Value of patch tests in clindamycin-related drug eruptions. Contact Dermatitis. $2011 ; 65: 202-7$.

26. Gaig P, Garcia-Ortega P, Baltasar M, Bartra J. Drug neosensitization during anticonvulsant hypersensitivity syndrome. J Investig Allergol Clin Immunol.2006;16:321-6.

27. Fredi NB, Aouam K, Chaabane A, Toumi A, Ben Rhomdhane $F$, et al. Hypersensitivity to amoxicillin after drug rash with eosinophilia and systemic symptoms (DRESS) to carbamazepine and allopurinol: a possible co-sensitization. Br J Clin Pharmacol. 2010;70:273-6.

28. Romano A, Viola M, Gaeta F, Rumi G, Maggioletti $M$. Patch testing in non-immediate drug eruptions. Allergy Asthma Clin Immunol. 2008;4:66-74. 